\title{
Pregnant women predisposed to urinary tract infections: individual and social vulnerabilities.
}

\author{
Arnildo Korb ${ }^{1 *}$, Saionara Vitoria Barimacker², Maria Sabrina Telch dos Santos ${ }^{2}$, Mariana Sbeghen \\ Menegatti $^{2}$, Carine Vendruscolo ${ }^{3}$, Danielle Bezerra Cabral ${ }^{3}$ \\ ${ }^{1}$ Agroveterinary Sciences Center, State University of Santa Catarina, Florianopolis, Santa Catarina, Brazil \\ ${ }^{2}$ Department of Nursing in Primary Health Care, State University of Santa Catarina, Florianopolis, Santa Catarina, \\ Brazil \\ ${ }^{3}$ Department of Vegetal Biology, State University of Santa Catarina, Florianopolis, Santa Catarina, Brazil
}

\begin{abstract}
Objectives: Diagnose individual and social vulnerabilities that predispose pregnant women to urinary tract infections (UTI). Method: 92 pregnant women were interviewed treated by the Brazilian public health service. Results: Individual vulnerabilities: 4 (4.0\%) were younger than 15 years, $6(6.0 \%)$ more than $35.47(51 \%)$ developed the infection, $11(12 \%)$ had anal intercourse, $9(82 \%)$ of these developed UTI; $16(17.3 \%)$ had oral sexual intercourse, of which 12 (75\%) developed UTI. Social vulnerabilities: $45(51.7 \%)$ had income between US\$ 298 and US\$ 596. Only $66(72.0 \%)$ finished high school. Of the 15 pregnant women with incomplete primary education, 11 (73.33\%) developed UTI. Conclusion: These vulnerabilities influenced the development of the infection.
\end{abstract}

Keywords: Urinary tract infections, Pregnant women, Health vulnerability.

Accepted on August 18, 2018

\section{Introduction}

Among the dimensions of vulnerability, individual, social and pragmatic ones, are considered relevant to public health. This study will focus on analysing the first two mentioned in a group of pregnant women and their predispositions regarding the development of infections. For this, individual vulnerability is understood as attitudes to protective practices anchored in intersubjective relationships, that is, behavioural habits of individuals, information and knowledge about specific situations. In the social dimension, contextual aspects such as social exclusion, economic relations, gender, ethnic/racial, religious beliefs and others are based on their definition [1].

Therefore, there are a number of gestational conditions that contribute to UTI susceptibility, such as increased bladder volume and a decreased tonus detrusor muscle, as well as ureteral dilation by relaxation of the smooth muscle and gravid uterus, causing hydro nephrosis due to the presence of progesterone. There is also an association of asymptomatic bacteriuria in pregnant women (pyelonephritis, cystitis) and the foetus (low birth weight, perinatal mortality and preterm labour). Studies in pregnant women describe rates of $28 \%$ of pyelonephritis and $12.8 \%$ of pre-term UTI [2]. In addition, the Ministry of Health proposes an operational classification of reproductive risk factors in which individual characteristics and unfavourable sociodemographic conditions are highlighted. Among these conditions would be schooling level, family or marital conflicts, unfavourable working conditions, exposure to unhealthy environments, with inequalities and discriminations by social class, gender, skin colour, age, among others. The objective of this study was to identify, in pregnant women attended by the single health system, individual and social vulnerabilities could predispose them to the development of UTI.

\section{Materials and Methods}

Pregnant women enrolled in the System for the Follow-up of the Prenatal and Birth Humanization Program (SispreNatal) in the municipality of Chapeco, SC, were interviewed in the health centers of Efapi and Sao Pedro neighbourhoods. These districts have, historically, different socioeconomic characteristics. Those eligible for this study were pregnant women who undertook the first prenatal visit between November 1, 2015, and February 29, 2016.

The average follow-up time attributed to each gestation was seven months, since in Brazil the pregnant women in the public health system have, for the most part, the first obstetric visit from the second month of gestation. A questionnaire with a pre- established questionnaire about sociodemographic conditions and knowledge about UTI (clinical manifestations, complications, mode of transmission, sexual practice and hygiene).

Four categories, A, B, C and D, were established to classify the age of the interviewed pregnant women. In these, A (less than 15 years), B (16 to 19 years), C (20 to 34 years) and D (35 years or more)

The project complied with Resolution 466/2012 of the National Health Council of Brazil and had approval in the 1,299,379 of October 28, 2015, by the Committee of Ethics in Research in Human Beings of UDESC. 
Citation: Korb A, Barimacker SV, Santos MST, et al. Pregnant women predisposed to urinary tract infections: individual and social vulnerabilities. J Preg Neonatal Med 2018;2(2):31-33.

\section{Results}

Of the 92, 47 (51.07\%) developed UTI in gestation in the development of the research (Table 1).

Table 1. Socioeconomic conditions and factors predicting to the development of UTI according to the age categories ( $A, B, C$ and $D)$.

\begin{tabular}{|l|}
\hline Legend \\
\hline$A^{*}, B^{*}, C^{*}$ and $D *=$ Groups $A, B, C$ and $D$ \\
\hline I.E.S ${ }^{*}=$ Incomplete elementary school \\
\hline C.E.S ${ }^{*}=$ Complete Elementary School \\
\hline I.H.S ${ }^{*}=$ Incomplete High School \\
\hline C.H.S ${ }^{*}=$ Complete High School \\
\hline I.H.E ${ }^{*}=$ Incomplete higher education \\
\hline C.H.E ${ }^{*}=$ Complete higher education; \\
\hline SI ${ }^{*}=$ Sexual intercourse \\
\hline
\end{tabular}

Of the 92 interviewees, $11(12 \%)$ reported having anal intercourse. Of these, 9 (82\%) developed UTI. Sixteen $(17.3 \%)$ reported oral sexual intercourse. Of these 16, $12(75 \%)$ developed UTI.

The highest prevalence of UTI was observed in primary education. Of the 38 pregnant women 20 (52.82\%) developed UTIs, $11(73.33 \%)$ of these had only incomplete elementary education. However, of the $21(23.1 \%)$ pregnant women in high school, only 9 (42.8\%) developed the infection. Low schooling is one of the obstacles to the effectiveness of health promotion actions (3). So much so that, of the 9 smokers (all of category C), $6(66.6 \%)$ had only attended primary education, and $4(44.4 \%)$ of these developed UTI. Of the $10(10.8 \%)$ who consumed alcoholic beverages, 4 (40\%) developed the infection.

The study showed that pregnant women under the age of 15 years old $n=4(4.0 \%)$ are socioeconomically vulnerable, since pregnancy in this age group makes it difficult to follow the sequence of educational training, especially in the acquisition of knowledge relevant to vocational training. However, the higher incidence of ITU was found in category B, where of the 24 pregnant women, $15(62.5 \%)$ developed UTI, whereas in category $\mathrm{C}$, of the 60 pregnant women, 28 (46.6\%) developed the infection. In this context, younger and sexually active women are more vulnerable to this clinical condition [2].

It was identified that low income, one to two minimum wages (US \$ 294 and US \$ 588), together with low educational level, predisposes the occurrence of UTI. Our findings highlight that of the 45 pregnant women who received up to US \$588, 26 $(57.7 \%)$ developed infection. In the group of 25 pregnant women who received between US \$ 588 and US \$ 882, 13 $(52 \%)$ had UTI, whereas in the group of 17 who received between US \$ 882 and US \$2,940, only $6(35.2 \%)$ had infections.
Of the 45 married women, only $17(37.7 \%)$ developed an infection, while among the 11 who reported having partners, 6 (54\%) had UTI, and 21 (63.6\%) had stable UTI. We did not investigate the relations of coexistence of stable union between the interviewees. Living with the partner most often increases family income and improves the conditions of access to health services, such as the inclusion of the partner in prenatal consultations [3]. Single, black, low-income, and educated women had less than six prenatal visits, no urine tests as recommended. This fact predisposes the occurrence of asymptomatic bacteriuria and late diagnosis of urinary tract infections [4].

Some sexual habits, such as anal and oral intercourse, may increase the risk of UTI susceptibility due to the contamination of the woman's urinary system. During the sexual act, microorganisms found in the microbiota of the human intestine are transported upwardly from the outside of the anal region to the woman's urinary system, triggering the infectious process. Of the 92 interviewees, 3 (3.2\%) maintained daily relationships and all of them developed infection. However, of the 15 pregnant women who did not have sexual intercourse in this period, 9 (60\%) had UTI. Could these, perhaps, belong to some of the vulnerable groups, such as the low income and without a fixed partner.

Of the 71 pregnant women who reported to perform intimate hygiene before sexual intercourse, 35 (49.2\%) developed UTI, while $9(77.7 \%)$ did not. Of the $84(91.3 \%)$ who performed hygiene after the relationships, $42(50 \%)$ had UTI. What needs to be ensured is the quality of this hygiene and the highest water intake in order to reduce Susceptibility to UTI [5].

The majority (98\%) declared to have garbage collection and treated water in the residence. However, $68 \%$ had no sewage system. This population is that of the Efapi district, which does not receive this service. This sewage is despised in septic tanks, sinkhole style.

Although, in most of the analyses performed in this study, the results for the development of UTI remained similar among the analysed variables, approximately $50 \%$, data from those of other studies (around 30 to 40\%). The results showed, therefore, that social and individual vulnerability conditions influence, in most situations, the development of this infection in pregnant women.

\section{References}

1. Ayres JR, Calazans GJ, Saletti FHC, et al. In: Collective Health Treaty. Risk, vulnerability and practices of prevention and health promotion. 2006; pp 375-417.

2. Foxman B. Urinary tract infection syndromes: occurrence, recurrence, bacteriology, risk factors, and disease burden. Infect Dis Clin N Am. 2014;28:1-13.

3. August SL, Rosa MJ. Evaluation of the prevalence of urinary tract infection in rural Panamanian women. PLoS One. 2012;7:e47752. 
4. Silveira MF, Barros AJD, Santos IS, et al. Socioeconomic differentials in performing urinalysis during prenatal care. Rev Saude Publica. 2008;42:389-95.

5. Chenoweth C, Saint S. Preventing catheter-associated urinary tract infections in the intensive care unit. Crit Care Clin. 2013;29:19-32.

\section{*Correspondence to}

Arnildo Korb

Catarina Agroveterinary Sciences Center

State University of Santa

Brazil

E-mail: arkorb@yahoo.com.br 